\title{
Valoración de la conducta en ratas Sprague Dawley sometidas a enriquecimiento ambiental
}

\author{
Madrid, Alanna \\ INDICASAT AIP \\ Panamá, Panamá \\ amadrid@indicasat.org.pa \\ Rivera, René \\ INDICASAT AIP \\ Panamá, Panamá \\ rrivera@indicasat.org.pa \\ Herrera, Lizzi \\ INDICASAT AIP \\ Panamá, Panamá \\ lherreram@indicasat.org.pa \\ Carreira, María \\ INDICASAT AIP \\ Panamá, Panamá \\ 0000-0001-5306-0021 \\ Britton, Gabrielle \\ INDICASAT AIP \\ Panamá, Panamá \\ https://orcid.org/0000-0002-1758-2495 \\ De Jesús, Rosa \\ INDICASAT AIP \\ Panamá, Panamá \\ https://orcid.org/0000-0001-9670-291X
}

\section{Abstract}

Routine procedures, such as restraint, substance administration, or blood collection, can produce an acute stress response in laboratory animals. The objective of this study was to determine if environmental enrichment in the housing boxstimulate anxiolytic behavior after an aversive procedure in Sprague Dawley rats of the INDICASAT AIP animal facility. At time 
of weaning two groups were formed: one with environmental enrichment and another in standard housing conditions without environmental enrichment. Two (2) polyvinylchloride tubes per cage were used as enrichment devices. After eight weeks, animal behavior was evaluated in the open field and elevated plus maze before and after an aversive procedure. The aversive procedure consisted of a retroorbital blood draw after mild sedation with isofluorane. The parameters evaluated in the open field, such as mobility and immobility, did not show significant differences between the groups housed in the enriched environment and the non-enriched environment. In relation to elevated plus maze behavior, animals housed in enriched environments spent more time in the open arms ( $35 \pm 21 \mathrm{~s})$ than those without enrichment $(7 \pm 6 \mathrm{~s})$. These data support the use of environmental enrichment to significantly reduce anxiety in rats after being subjected to an aversive procedure.

Keywords: rats, environmental enrichment, anxiolytic behavior.

\section{Resumen}

En los animales de laboratorio, se conoce que los procedimientos de rutina como la manipulación, la sujeción, las inyecciones o la toma de muestras de sangre provocan una respuesta aguda de estrés. El objetivo de este ensayo fue determinar si la condición de enriquecimiento del alojamiento de las ratas Sprague Dawley producidas en el Bioterio de INDICASAT-AIP, puede favorecer una conducta ansiolítica luego de un procedimiento aversivo como la toma de muestra de sangre. Al destete, los animales se segregaron en 2 grupos que incluyeron: grupo con y sin enriquecimiento. El enriquecimiento ambiental consistió en 2 tubos de policloruro de vinilo por caja. Ocho semanas después, los animales se sometieron a las pruebas de conducta de campo abierto y laberinto en cruz elevado antes y después de un procedimiento de toma de muestra sanguínea vía retroorbital previa sedación con isofluorano. En los parámetros valorados en campo abierto: tiempo de movilidad e inmovilidad, no se observó un efecto significativo entre los grupos con enriquecimiento y sin enriquecimiento. En relación a los parámetros del laberinto en cruz elevado, se encontró que los animales alojados en el ambiente con enriquecimiento pasaron en promedio una mayor cantidad de tiempo ( $35 \pm 21 \mathrm{~s}$ ), en brazos abiertos, que el grupo sin enriquecimiento ( $7 \pm 6 \mathrm{~s})$. Estos datos permitieron comprobar que el enriquecimiento ayudó a disminuir la ansiedad en las ratas del ensayo, luego de ser sometidas a un procedimiento aversivo como la toma de muestra sanguínea.

Palabras claves: ratas, enriquecimiento ambiental, conducta ansiolítica. 


\section{INTRODUCCIÓN}

Las condiciones mínimas de alojamiento de ratas y ratones son dictadas por la Guía para el Cuidado y Uso de Animales de Laboratorio [1] en esta se recomienda la mejora de los entornos estructuralmente y socialmente para promocionar el comportamiento típico de la especie que sea usada en la investigación, mejorando el bienestar animal. Se considera que el enriquecimiento ambiental es útil para una amplia gama de experimentos que estudian los efectos del medio ambiente en los ratones. Se ha reportado, igualmente la influencia de este en datos de experimentos tanto de cáncer como de metabolismo [2].

En los animales de laboratorio, procedimientos de rutina tales como la manipulación, la sujeción, las inyecciones o la toma de muestras de sangre provocan una respuesta aguda de estrés, por lo que condiciones que minimizen esta respuesta, como el enriquecimiento ambiental, son considerados como una contribución al bienestar de los animales [3] Los modelos existentes para la evaluación ansiolítica en animales han tomado un gran auge en los últimos tiempos debido a la estrecha relación que existe en el comportamiento humano, convirtiéndose en un peldaño para la elaboración de fármacos o soluciones naturales que permitan controlar ciertos factores de estrés, miedo, ansiedad [4]. El evaluar si el enriquecimiento ambiental tiene algún efecto sobre parámetros conductuales de ansiedad, conllevaría utilizar enriquecimiento en los alojamientos de los animales para promover un estadio ansiolítico en las ratas Sprague Dawley de ambos sexos, ayudando al bienestar del animal.

El ensayo se realizó en la unidad de producción y experimentación de producción animal del INDICASAT-AIP, con la finalidad de dar a conocer a los investigadores usuarios lo favorable o no de que los animales que usan en sus investigaciones cuenten con enriquecimiento ambiental.

\section{MÉTODO}

Este trabajo contó con el aval del CICUA - INDICASAT bajo el número CICUA- 20-001.

\section{A. Animales usados}

Se usaron ratas hembras y machos, de la línea no consanguínea Sprague Dawley. Producidos y mantenidos en la unidad de producción y experimentación de animales del INDICASAT AIP. Estos son mantenidos en condiciones de alta barrera sanitaria. Alojados en cubículos con temperatura ambiental de $18^{\circ} \mathrm{C}$, Humedad Relativa $65 \%$, con ciclos de 12 horas luz:12 horas oscuridad. Al destete se establecieron los grupos: con enriquecimiento (CE), y sin enriquecimiento (SE). 


\section{B. Parámetros evaluados}

Se valoraron en campo abierto: tiempo de movilidad e inmovilidad, número de entradas a la zona central, tiempo en el centro del campo abierto.

En relación a los parámetros del laberinto en cruz elevado se valoraron: la cantidad de tiempo que se pasan en los brazos abiertos (TBA) y la cantidad de tiempo que se pasan en los brazos cerrados (TBC).

\section{DISEÑO EXPERIMENTAL}

Al destete los animales se agruparon en dos grupos experimentales, uno al cual se alojó con enriquecimiento ambiental, que consistió en 2 tubos de polivinil cloruro, de aproximadamente $20 \mathrm{~cm}$ de largo por 8 pulg. cada uno. A las 8 semanas se les realizó una toma de muestra de sangre vía retroorbital previa sedación con isofluorano $(100 \%$ en cámara de vidrio), comprobada que no tenían ninguna respuesta a estímulos podales de las cuatro extremidades. Se procedió a realizar la toma de muestra retroorbital, como procedimiento aversivo. Posteriormente los animales se dejan recuperarse de la anestesia y se devuelven a sus alojamientos.

Se valoró la conducta dos días previos a la toma de muestra, 2 horas previas a la toma de muestra y una hora luego de la toma de muestra sanguínea. Estas se colocaron en campo abierto, ya que teniendo en cuenta las características naturales de las ratas, el campo abierto (particularmente cuando está fuertemente iluminado) constituye para la rata un medio adverso (nivel medio o moderado de estrés) lo que inducirá en el animal un aumento de emotividad, aumento que suele traducirse en una pérdida de su capacidad exploratoria. Además, se debe valorar de manera distinta los desplazamientos en zonas próximas a la pared (deambulación externa) de aquellos realizados en zonas alejadas (deambulación interna), ya que, presumiblemente, la respuesta tigmotáxica sólo se inhibe cuando el nivel emotivo del animal es bajo y se aventura a desplazarse hacia el centro del recinto.

Dos días luego de la toma de muestra se valoró el comportamiento de las ratas en el laberinto en cruz elevado, con la finalidad de registrar el posible efecto de ansiedad que podían tener los animales que eran sometidos al procedimiento experimental del sangrado considerando que los animales con menos entradas a los brazos abiertos, así como la locomoción reducida reflejarán niveles altos de ansiedad.

\section{RESULTADOS}

En relación al campo abierto no se observaron diferencias significativas entre la conducta de los animales alojados con enriquecimiento ambiental y los que no contaron con enriquecimiento. 
En relación al comportamiento en el laberinto en cruz elevado se observó que los animales que se alojaron en ambiente con enriquecimiento pasaron más tiempo en los brazos abierto (Figura 1), en comparación con los que se alojaron en un ambiente sin enriquecimiento (ce $=35 \pm 21 \mathrm{~s}$ vs $\mathrm{SE}=7 \pm 6 \mathrm{~s}$ ).

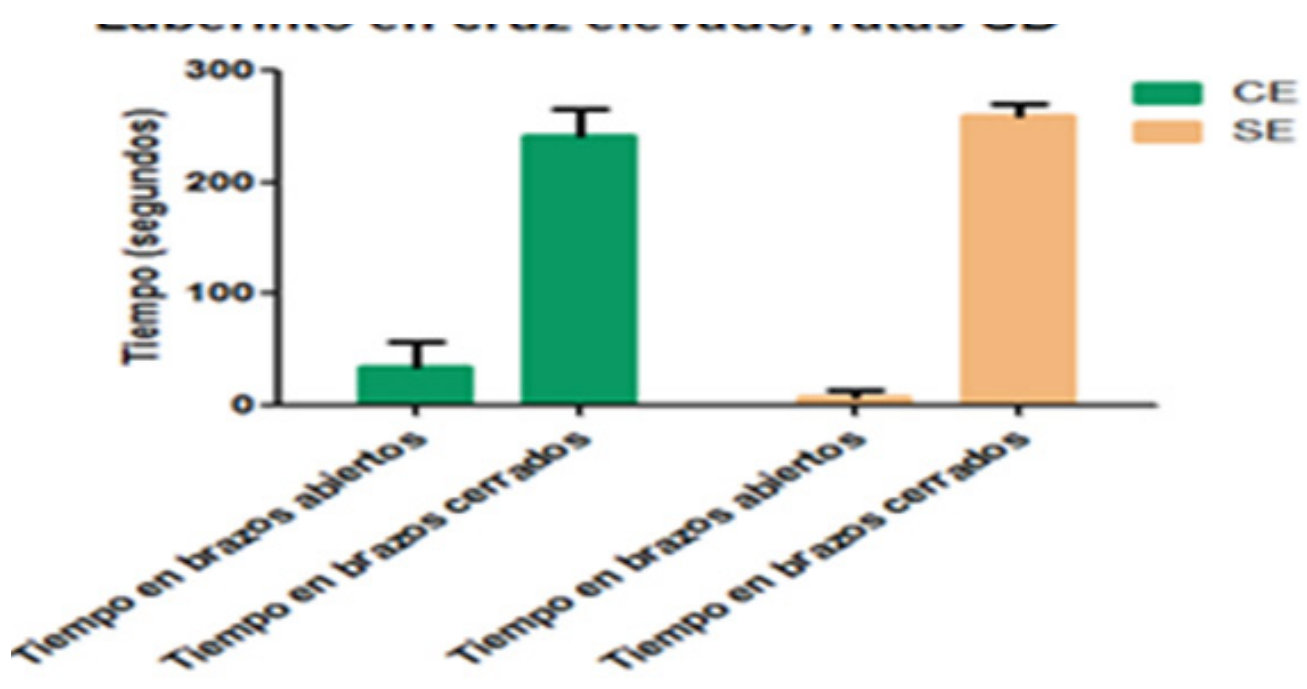

Figura 1. Comportamiento de las ratas en el laberinto en cruz elevado.

\section{CONCLUSIÓN}

Los resultados permiten concluir que existe un efecto significativo del enriquecimiento ambiental sobre la ansiedad de los animales cuando son sometido a un procedimiento aversivo. El uso de enriquecimiento ambiental contribuye a ameliorar la respuesta de estrés y ansiedad, contribuyendo al bienestar de los animales de laboratorio.

\section{Referencias}

[1] [1] Nathional Research Council. Guide for the care and use of Laboratory Animals. Washington. p. 209. 2011.

[2] [2] L. Cao, E. Choi, X. Liu, A. Martin, Ch Wang, X. Xu, M. During. White to brown fat phenotypic switch induced by genetic and environmental activation of a hypothalamic-adipocyte axis. Cell Metab. 14(3). p. 324-338. 2011.

[3] [3] Meijer, M. K., Sommer, R., Spruijt, B. M., van Zutphen, L. F. M., Baumans, V., \& Department of Animals, Science \& Society. Influence of environmental enrichment and handling on the acute stress response in individually housed mice. Laboratory Animals, (41), pp:161-173. 2007.

[4]

[5] [4] D. Moreno, J. Pazmiño, M. Guerra, G. Pilco, M, Zabala. Implementación de sistemas evaluadores de conducta ansiolítica en ratones de laboratorio utilizando procesamiento digital de imágenes. Maskay vol.8 no.1 pp.27-34. 2018. 


\section{Autorización y Licencia CC}

Los autores autorizan a APANAC XVIII a publicar el artículo en las actas de la conferencia en Acceso Abierto (Open Access) en diversos formatos digitales (PDF, HTML, EPUB) e integrarlos en diversas plataformas online como repositorios y bases de datos bajo la licencia CC:

Attribution-NonCommercial-ShareAlike 4.0 International (CC BY-NC-SA 4.0) https://creativecommons. org/licenses/by-nc-sa/4.0/.

Ni APANAC XVIII ni los editores son responsables ni del contenido ni de las implicaciones de lo expresado en el artículo. 